Produto \& Produção, vol. 11, n. 1, p. 22 - 29, fev. 2010

Edição Metrologia

\title{
Aplicação da Calorimetria Exploratória Diferencial (DSC) para Determinação da Pureza de Fármacos
}

Gabriela F. Moreira, D. Sc gfmoreira@inmetro.gov.br

Andrea Balbo, D. Sc

Carlos A. Achete, Prof. caachete@inmetro.gov.br

Instituto Nacional de Metrologia , Normalização e Qualidade Industrial - INMETRO Diretoria de Metrologia Científica e Industrial Divisão de Metrologia de Materiais

Juliano S. Barin, D. Sc

Wagner Wollinger, M. Sc wwollinger@inmetro.gov.br

Raquel Nogueira, D. Sc

Celso F. Bittencourt, D. Sc

Janaína M. Rodrigues, D. Sc

Instituto Nacional de Metrologia , Normalização e Qualidade Industrial - INMETRO Diretoria de Metrologia Científica e Industrial Divisão de Metrologia Química

\section{RESUMO}

O trabalho aborda um estudo comparativo entre a técnica de Cromatografia a Líquido de Alta Eficiência e a técnica de Calorimetria Exploratória Diferencial, visando avaliar a aplicação desta para a determinação direta da pureza de fármacos candidatos a materiais de referência certificados.

Palavras-chave: DSC, pureza, fármacos. 
A qualidade dos medicamentos constitui um fator fundamental para assegurar a sua eficácia e minimizar a ocorrência de efeitos indesejados decorrentes da presença de impurezas e/ou produtos de degradação. Para tanto, diversos procedimentos são preconizados em compêndios oficiais, tais como as Farmacopeias, para determinar a pureza das matérias-primas utilizadas na fabricação das diversas formas farmacêuticas. Assim, pode-se afirmar que a determinação da pureza dos princípios ativos é essencial e consiste em atividade laboratorial rotineira no controle de qualidade de medicamentos.

Para este fim, várias técnicas analíticas têm sido recomendadas nas Farmacopeias, envolvendo métodos clássicos e métodos instrumentais. Nos últimos anos, percebeu-se um aumento considerável da utilização dos métodos instrumentais, especialmente da cromatografia a líquido de alta eficiência (CLAE) (SANDOR GÖROG, 2005).

Entretanto, os métodos farmacopeicos para a determinação da pureza de fármacos por CLAE demandam a utilização de quantidades significativas dos materiais de referência, tanto dos princípios ativos quanto de suas impurezas, o que torna o procedimento caro e demorado. Convém ainda lembrar que padrões de impurezas nem sempre se encontram disponíveis.

Desta forma, a utilização de uma técnica que permita a determinação direta da pureza de fármacos evitando o uso demasiado de materiais de referência consistiria em uma alternativa interessante para o controle de qualidade de medicamentos. Para tanto, dentre 0 arsenal analítico disponível atualmente, a Calorimetria Exploratória Diferencial (DSC) apresenta-se bastante atraente. Além de não envolver a utilização de materiais de referência para a determinação da pureza, apresenta outras vantagens, tais como menor tempo de análise e de preparo da amostra (MATHKAR, 2009).

A análise da pureza por DSC é uma técnica bem consolidada, sendo que a metodologia empregada está descrita na Norma ASTM E 928-03 ("Standard test method for purity by differential scanning calorimetry"). O método avalia a pureza do composto por meio de uma análise do pico de fusão obtido, aplicando a lei da depreciação do ponto de fusão de Van't Hoff (que prevê a depreciação do ponto de fusão do composto puro devido à presença de impurezas) (A VAN DOOREN, 1984).

A lei assume algumas considerações, e dessa forma, esta somente é válida quando: o material fundido é uma solução ideal na qual as impurezas são solúveis; o conteúdo das impurezas é inferior a 2,5\% mol; o processo de fusão ocorre em condições de quase-equilíbrio termodinâmico; a capacidade térmica do sólido é igual à do líquido; as impurezas são insolúveis no estado sólido; o composto não se decompõe ou não reage com atmosfera e/ou com as impurezas; não existem transições próximas da temperatura de fusão; a entalpia de fusão é independente da temperatura; o sólido é totalmente cristalino (A VAN DOOREN, 1984).

Assim, dentro deste contexto, o presente trabalho busca avaliar a utilização do método acima descrito, que emprega o DSC, na determinação da pureza de três substâncias distintas candidatas a material de referência certificado (MRC); entre eles captopril, diclofenaco sódico e diclofenaco potássico. Os resultados foram 
comparados com aqueles obtidos por CLAE conjuntamente com o método de Karl Fischer e titulação.

\section{OBJETIVO}

Avaliar a aplicação da técnica de Calorimetria Exploratória Diferencial (DSC) na determinação da pureza de fármacos candidatos a materiais de referência certificados.

\section{MÉTODOS}

Materiais - as amostras de captopril, diclofenaco sódico e diclofenaco potássico foram obtidas diretamente de fornecedores de matéria-prima para a indústria farmacêutica.

Calorimetria Exploratória Diferencial (DSC) - as análises de DSC foram conduzidas utilizando o equipamento DSC Q1000 da TA Instruments, com o acessório de refrigeração RCS. Antes de iniciar os ensaios, O DSC Q1000 inicialmente foi calibrado utilizando padrões de safira, fornecido pelo fabricante, para calibração da linha base do equipamento, com correção automática do "atraso térmico" associado ao fluxo de calor. Após, para a calibração da constante da célula do equipamento e da temperatura, foram utilizados padrões de índio e bismuto, materiais de referência rasteáveis certificados pelo NIST.

As amostras foram analisadas em panelas de alumínio herméticas (TA Instruments) fechadas e para cada análise foram utilizados 1 a $3 \mathrm{mg}$ de material (determinados em uma balança analítica, marca Mettler, modelo UMX5). As análises foram realizadas em triplicada aquecendo a amostra com uma taxa de aquecimento de $0,5^{\circ} \mathrm{C} / \mathrm{min}$, até atingir a temperatura de $10-15{ }^{\circ} \mathrm{C}$ acima de sua temperatura de fusão, sob fluxo de He (50 mL/min).

A metodologia utilizada para a análise da pureza é descrita pela Norma ASTM E 928-03.

A determinação da pureza pela técnica de DSC aplica a lei da depreciação do ponto de fusão, ocasionada pela presença de impurezas, de Van't Hoff, que está representada na Equação 1.

$$
\mathrm{T}_{S}=\mathrm{T}_{0}-\frac{R T_{0}^{2} \chi}{\Delta H} \frac{1}{\mathrm{~F}}
$$

Na qual: TS é a temperatura observada da amostra; T0 é a temperatura de fusão da amostra pura (100\%); $\chi$ é a fração molar das impurezas; $\Delta \mathrm{H}$ é o calor de fusão da amostra pura, e F é fração de material fundido, que é determinada através da medida das áreas parciais do pico de fusão experimental.

A equação anterior pode ser considerada de primeiro grau assumindo a forma apresentada na Equação 2:

$Y=a X+b$

onde: 


$$
X=\frac{1}{F}, \quad Y=T_{s}, \quad a=-\frac{R T_{0}^{2} \chi}{\Delta H} \quad \text { e } \quad b=T_{0}
$$

Normalmente, o gráfico obtido de TS versus 1/F não é linear. A linearização é realizada acrescentando uma área arbitrária (c) à área total do pico, e a cada área parcial, conforme mostra a Equação 3.

$$
F=\frac{A_{\text {part }}+c}{A_{\text {tot }}+c}
$$

Na qual: Apart é a área parcial do pico que representa a fração da amostra fundida; Atot é a área total do pico de fusão e c é a área adicional.

A norma apresenta dois métodos para o cálculo da pureza do composto a partir da equação de Van`t Hoff acima apresentada. Sendo que o método empregado no trabalho foi o "Método A", que utiliza para o cálculo o Software TA Universal Analysis 2000 desenvolvido pela TA Instruments.

O software faz a análise dos dados adquiridos, detecta a linha de base, a área e a altura máxima do pico de fusão. Estes dados são utilizados para o cálculo dos valores e números de áreas parciais, onde o software divide automaticamente a curva experimental. Sendo que para análise do pico foram usadas 50 áreas parciais, que tem uma fração máxima permitida de $50 \%$, sendo o valor da altura de corte da primeira área parcial de 0,01\%.

O valor de c é determinado utilizando a técnica não linear de mínimos quadrados. Assim, determinado c, é feita a linearização da curva TS versus 1/F. Após, efetua-se o cálculo do coeficiente linear e angular da reta aplicando uma regressão linear. A partir do coeficiente angular determina-se a fração molar das impurezas presentes em cada amostra.

As análises foram realizadas em triplicata. As incertezas expandidas foram calculadas com fator de abrangência 2,0 correspondentes a 95\% de nível de confiança.

Cromatografia a líquido de alta eficiência (CLAE) e Karl Fischer - a pureza do composto captopril foi determinada pelo método indireto de balanço de massas, em que os resultados das impurezas são descontados da unidade. Foi utilizada CLAE para análise das impurezas orgânicas e titulação pelo método de Karl Fischer para análise de água. A CLAE foi realizada com detector de arranjo de fotodiodos (DAD) em sistema Shimadzu LC-20, programa LC Solution e colunas C18 (Supelcosil LC$18250 \times 4,6 \mathrm{~mm}, 5 \mu \mathrm{m}$ ) e nitrila (Supelcosil LC-CN $150 \times 4,6 \mathrm{~mm}, 5 \mu \mathrm{m}$ ).

As amostras foram preparadas em triplicata por gravimetria, sendo injetadas 3 vezes cada amostra. Em cada caso, foram preparadas soluções concentradas (500 $\mu \mathrm{g} / \mathrm{g}$ para captopril) e diluídas (2 e $10 \mu \mathrm{g} / \mathrm{g}$, respectivamente). A concentração de impurezas orgânicas foi determinada pela razão entre as áreas dos picos de cada impureza na solução concentrada e a área do pico de captopril na solução diluída, devidamente corrigida pelo fator de diluição (razão entre as concentrações das soluções concentrada e diluída). As incertezas expandidas foram calculadas com fator de abrangência em torno de 2, correspondente a $95 \%$ de nível de confiança.

A determinação da água das amostras foi feita pelo método de Karl Fischer em equipamento Metrohm titulador coulométrico. As impurezas inorgânicas do captopril foram determinadas por resíduo de ignição segundo Farmacopeia 
Brasileira. Componentes voláteis foram avaliados pelo teste de perda por dessecação segundo Farmacopeia Brasileira.

Titulação - o teor de pureza do diclofenaco sódico e do diclofenaco potássico foi determinado através de estudo interlaboratorial, de acordo com as recomendações da OMS, utilizando-se volumetria de oxi-redução em meio não aquoso com ponto final potenciométrico. O material foi ainda caracterizado de acordo com Farmacopeia Brasileira.

\section{RESULTADOS E DISCUSSÃO}

A Figura 1 ilustra o gráfico de DSC adquirido para o composto captopril e os valores encontrados a partir do cálculo descrito na metodologia e empregado na quantificação do teor de pureza do material.

Como pode ser observado na Figura 1, foi verificado durante o clico de aquecimento do captopril um pico endotérmico bem definido e simétrico, característico de transição de fase da fusão e que denota uma transição a temperatura constante, indicando que o processo ocorre no quase-equilíbrio termodinâmico. A linha base foi mantida, sugerindo também que a capacidade térmica do material não se modifica durante o processo. Ademais, não ocorre nenhuma outra transição de fase durante a varredura da temperatura. Sendo assim, o material apresentou condições ideais para efetuar-se o cálculo de sua pureza por meio de seu pico de fusão obtido através da técnica de DSC.

As Figuras 2 e 3 apresentam as curvas de DSC obtidos para as amostras de diclofenaco sódico e potássico, respectivamente. Como pode ser observado na Figura 2, o formato do pico de fusão do composto (pico endotérmico) sugere que o mesmo é instável próximo ao seu ponto de fusão, pois ocorrem dois eventos (banda dupla), seguidos de uma alta oxidação do material, o que por sua vez é indicado pelo intenso pico exotérmico logo após a fusão do composto. Já para o diclofenaco potássico (Figura 3), foi observada uma alta liberação de voláteis, indicada pelos vários picos endotérmicos e exotérmicos, o que sugere sua degradação durante a análise térmica.

Dessa forma, para evitar a oxidação do material devido à presença de oxigênio da atmosfera nas panelas utilizadas nas análises de DSC, as amostras, tanto de diclofenaco sódico e diclofenaco potássico, foram preparadas e hermeticamente fechadas em uma câmara de luvas com atmosfera inerte (N2) controlada, após foram secas em uma estufa à120 ${ }^{\circ} \mathrm{C}$.

Novos testes foram realizados, porém os mesmos resultados foram observados, confirmando que os eventos verificados durante a análise térmica das amostras correspondem à decomposição destas conjuntamente com a transição de fusão, visto que a temperatura de fusão destes compostos é extremamente elevada, entre $260-280{ }^{\circ} \mathrm{C}$.

Contudo, para estes casos, a análise de DSC não é apropriada para a determinação da pureza, visto que não é possível observar um pico de fusão bem definido, partir do qual é efetuado cálculo para estes compostos. 


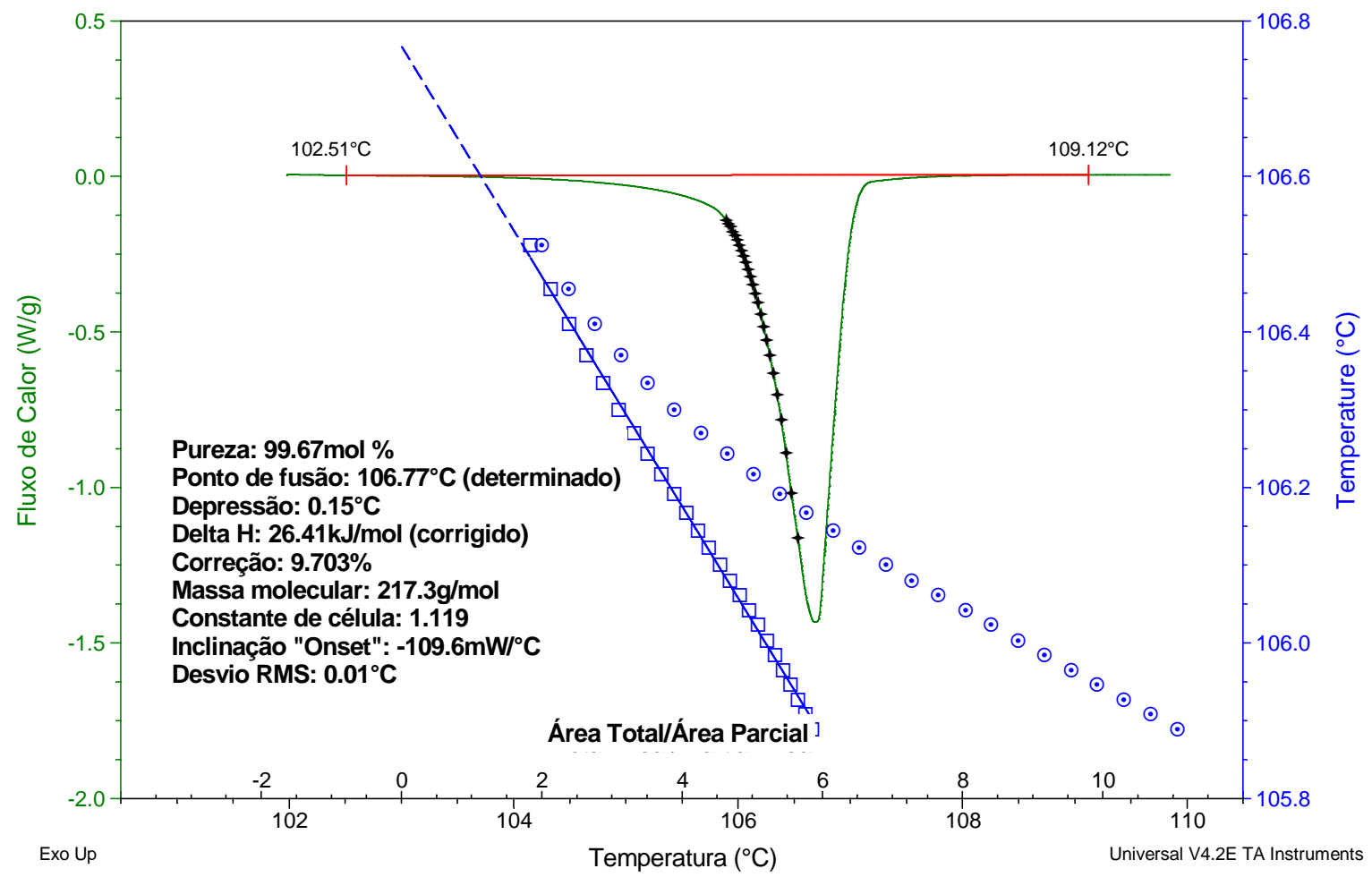

Figura 1 - Curva DSC obtida para a amostra de captopril na faixa de $100-115^{\circ} \mathrm{C}$ (taxa de aquecimento de $0,5^{\circ} \mathrm{C} / \mathrm{min}$, atm $\mathrm{He}$ ).

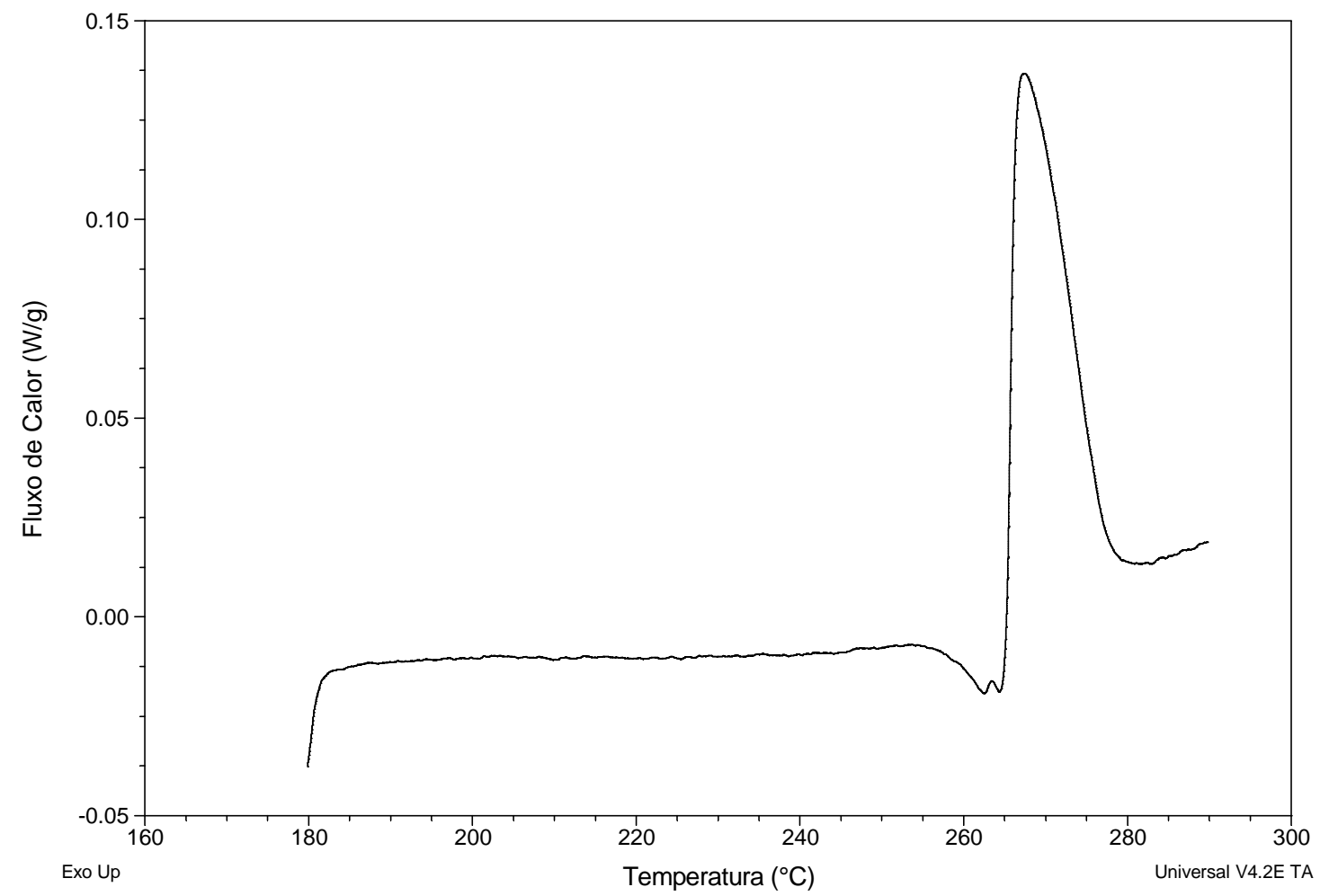

Figura 2 - Curva DSC obtida para a amostra de diclofenaco sódico na faixa de $180-290{ }^{\circ} \mathrm{C}$ (taxa de aquecimento de $0,5^{\circ} \mathrm{C} / \mathrm{min}$, atm $\mathrm{He}$ ). 


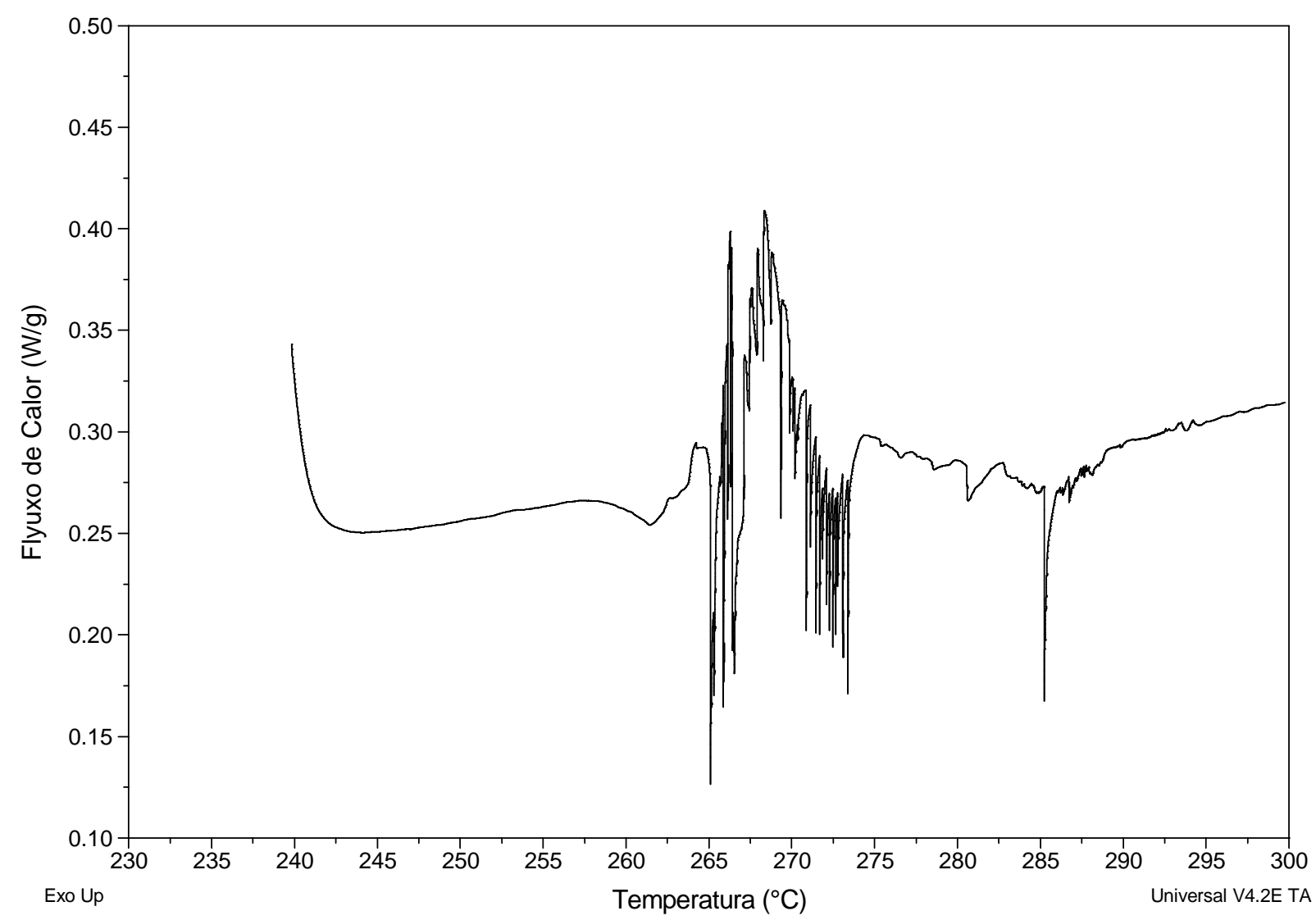

Figura 3 - Curva DSC obtida para a amostra de diclofenaco potássico na faixa de $235-300{ }^{\circ} \mathrm{C}$ (taxa de aquecimento de $0,5^{\circ} \mathrm{C} / \mathrm{min}$, atm $\mathrm{He}$ ).

A Tabela 1 apresenta os valores de pureza encontrados para o composto captopril a partir das técnicas de DSC e CLAE.

Os valores obtidos por DSC e por CLAE mostraram-se equivalentes, considerando a incerteza de cada medida. Além disso, a técnica de DSC apresentou uma elevada reprodutibilidade e precisão, conforme ilustrado pelos valores de incerteza obtidos (Tabela 1). Estes resultados, assim, evidenciam o potencial da técnica de DSC para a determinação de pureza de materiais.

Tabela 1 - Resultados obtidos para a determinação da pureza do captopril para técnicas de DSC e CLAE.

\begin{tabular}{|c|c|c|c|c|}
\hline & $\begin{array}{c}\text { DSC } \\
(\mathrm{mol} \%)\end{array}$ & & $\begin{array}{l}\mathrm{LAE} \\
\mathrm{m} / \mathrm{m})\end{array}$ & $\begin{array}{l}\text { CLAE } \\
(\mathrm{mol} \%)\end{array}$ \\
\hline & Média Incerteza* & Média & Incerteza* & Média \\
\hline Captopril & 99,650 & 99,66 & 0,33 & 99,83 \\
\hline $\begin{array}{c}\text { Diclofenaco sódico } \\
\text { Diclofenaco } \\
\text { potássico }\end{array}$ & \multicolumn{4}{|c|}{$\begin{array}{c}\text { Não foi possível realizar análise devido à degradação } \\
\text { térmica da amostra durante a análise térmica }\end{array}$} \\
\hline
\end{tabular}

*Nível de confiança de 95 \%, fator de abrangência em torno de 2,0 
A técnica de determinação de pureza por DSC é uma técnica direta, rápida e bastante precisa. Os resultados apresentados para a análise de captopril por DSC e CLAE demonstraram que, para princípios ativos cujo perfil de impurezas é conhecido, a pureza determinada por DSC mostra-se equivalente àquela determinada por CLAE, e, assim, o DSC mostra-se bastante atrativo. Porém, da mesma forma que acontece com as demais técnicas analíticas, a DSC também apresenta limitações, não podendo ser empregada para princípios ativos que se decompõem ao fundir (ex.: diclofenacos). Além disso, para princípios ativos cujo perfil de impurezas é desconhecido, os resultados da DSC podem diferir daqueles obtidos por CLAE.

\begin{abstract}
This work presents a comparative study between the High Performance Liquid Chromatography and Differential Scanning Calorimetry techniques as absolute methods of purity determination of materials regarding its application with pharmaceutical certificate reference materials.
\end{abstract}

Keywords: DSC, purity, pharmaceuticals.

\title{
Agradecimentos
}

Os autores agradecem ao apoio financeiro da FAPERJ e da FINEP.

\section{REFERÊNCIAS}

ASTM E 928-03. Standard Test Method for Purity by Differential Scanning Calorimetry", West Conshohocken, USA, 2003.

A VAN DOOREN, A et al. Purity determinations of drugs with differential scanning calorimetry (DSC) - a critical review. International Journal of Pharmaceutics v. 20, p. 217-233, 1984.

Farmacopeia Brasileira. 4. ed. São Paulo: Editora Atheneu. São Paulo, 1988.

MATHKAR, $\mathrm{S}$ et al. The use of differential scanning calorimetry for the purity verification of pharmaceutical reference standards. Journal of Pharmaceutical and Biomedical Analysis v. 49, n. 3, p. 627-631, 2009.

SANDOR GÖROG. The sacred cow: the questionable role of assay methods in characterizing the quality of bulk pharmaceuticals. Journal of Pharmaceutical and Biomedical Analysis,v. 36, n. 5, p. 931-937, 2005. 